\title{
Metabolism-related microRNAs in maternal breast milk are influenced by premature delivery
}

\author{
Molly C. Carney ${ }^{1}$, Andrij Tarasiuk ${ }^{1}$, Susan L. DiAngelo ${ }^{1}$, Patricia Silveyra ${ }^{1}$, Abigail Podany ${ }^{2}$, Leann L. Birch ${ }^{3}$, Ian M. Paul ${ }^{1}$,
} Shannon Kelleher ${ }^{4}$ and Steven D. Hicks ${ }^{1}$

BACKGROUND: Maternal breast milk (MBM) is enriched in microRNAs, factors that regulate protein translation throughout the human body. MBM from mothers of term and preterm infants differs in nutrient, hormone, and bioactive-factor composition, but the microRNA differences between these groups have not been compared. We hypothesized that gestational age at delivery influences microRNA in MBM, particularly microRNAs involved in immunologic and metabolic regulation.

METHODS: MBM from mothers of premature infants (pMBM) obtained 3-4 weeks post delivery was compared with MBM from mothers of term infants obtained at birth (tColostrum) and 3-4 weeks post delivery (tMBM). The microRNA profile in lipid and skim fractions of each sample was evaluated with high-throughput sequencing.

RESULTS: The expression profiles of nine microRNAs in lipid and skim pMBM differed from those in tMBM. Gene targets of these microRNAs were functionally related to elemental metabolism and lipid biosynthesis. The microRNA profile of tColostrum was also distinct from that of pMBM, but it clustered closely with tMBM. Twenty-one microRNAs correlated with gestational age demonstrated limited relationships with method of delivery, but not other maternal-infant factors.

CONCLUSION: Premature delivery results in a unique MBM microRNA profile with metabolic targets. This suggests that preterm milk may have adaptive functions for growth in premature infants.

M aternal breast milk (MBM) provides optimal nutrition for growth and development of infants. However, premature infants face a variety of physiological challenges that differ from infants born at term, and therefore they have different nutritional needs from maternal milk. When compared with the milk of mothers with term infants (tMBM), the milk of mothers with preterm infants (pMBM) has higher concentrations of protein, nitrogen, fat, phospholipids, and elements such as sodium, chloride, and iron $(1,2)$. Conversely, there are lower levels of lactose and zinc in $\mathrm{pMBM}$ compared with those in tMBM (1-3). Macronutrient profiles in MBM also vary with gestation of delivery. An example of this includes lipids: medium-chain fatty acids are higher in pMBM, but levels of oleic acid are lower (1). Analysis of the nutritional sufficiency of pMBM shows that nutrients received per volume of MBM exceed the intrauterine requirements for a given gestational age. In addition, preterm infants fed $\mathrm{pMBM}$ receive more nutrients than if they were fed tMBM $(1,2)$. The importance of these differences in $\mathrm{PMBM}$ and $\mathrm{TMBM}$ is highlighted by findings that preterm infants fed pMBM have improved health outcomes compared with the preterm infants fed tMBM (4).

Micro-ribonucleic acids (miRNAs) are non-coding nucleic acid sequences derived from inter- and intragenic DNA regions. They reduce the efficiency of gene expression, inhibiting protein translation through targeted degradation of mRNA transcripts. A single miRNA can bind to multiple mRNAs exponentially increasing its regulatory activity (5). Although miRNAs regulate numerous processes and are present in biofluids throughout the body, they are found in the greatest concentrations in MBM (6). The miRNAs found in $\mathrm{MBM}$ originate primarily from mammary gland epithelium, where they have a role in mammary epithelial cell function (7). A portion may also be derived from extramammary tissue via the bloodstream $(5,8,9)$. In MBM, miRNAs are packaged into vesicles such as exosomes (6). This accounts for the higher proportion of miRNAs in the lipid fraction of MBM compared with that in the skim fraction $(5,9)$. Recent studies have identified 281-602 unique miRNAs in human MBM, most of which are found in lipids $(5,8,10)$. Over $65 \%$ of MBM miRNAs are related to immune (10) or metabolic function (9,11-13). For example, miR-181a and miR-155 are abundant in breast milk and work together to increase B- and T-cell proliferation, resulting in enhanced adaptive immunity $(5,9,10)$. In addition, miR-33 and miR-122 (which are expressed in $\mathrm{MBM}$ ) regulate macromolecule synthesis via cholesterol and lipid homeostasis $(9,12)$.

\footnotetext{
${ }^{1}$ Department of Pediatrics, Penn State College of Medicine, Hershey, Pennsylvania; ${ }^{2}$ Department of Surgery, Penn State College of Medicine, Hershey, Pennsylvania;

${ }^{3}$ Department of Foods and Nutrition, University of Georgia, Athens, Georgia; ${ }^{4}$ Department of Cellular and Molecular Physiology, Penn State College of Medicine, Hershey, Pennsylvania. Correspondence: Steven D. Hicks (shicks1@hmc.psu.edu)

Received 21 November 2016; accepted 19 February 2017; advance online publication 24 May 2017. doi:10.1038/pr.2017.54
} 


\section{Premature birth and breast milk miRNA}

Table 1. Medical and demographic characteristics of maternal-infant dyads

\begin{tabular}{|c|c|c|c|c|c|c|c|c|}
\hline & \multicolumn{5}{|c|}{ Maternal characteristics } & \multicolumn{3}{|c|}{ Infant characteristics } \\
\hline & $\begin{array}{c}\text { Age } \\
\text { (years) }\end{array}$ & $\begin{array}{c}\text { Pre-pregnancy } \\
\text { BMI }\left(\mathrm{kg} / \mathrm{m}^{2}\right)\end{array}$ & $\begin{array}{l}\mathrm{BMI} \text { at time of } \\
\text { delivery }\left(\mathrm{kg} / \mathrm{m}^{2}\right)\end{array}$ & $\begin{array}{l}\text { White race/ } \\
\text { ethnicity (\%) }\end{array}$ & $\begin{array}{l}\text { Hypertension } \\
(\%)\end{array}$ & $\begin{array}{l}\text { C-section } \\
\text { delivery (\%) }\end{array}$ & $\begin{array}{l}\text { Gestational } \\
\text { age (weeks) }\end{array}$ & $\begin{array}{c}\text { Male } \\
(\%)\end{array}$ \\
\hline Term group $(n=13)$ & $32 \pm 4$ & $26 \pm 8$ & $34 \pm 12$ & 94 & 6 & 24 & $39 \pm 1$ & 47 \\
\hline Preterm group $(n=31)$ & $29 \pm 5$ & $\mathrm{~N} / \mathrm{A}$ & N/A & 86 & $43^{a}$ & $60^{a}$ & $32 \pm 3^{a}$ & 55 \\
\hline
\end{tabular}

N/A, not available.

a Denotes $P<0.05$.

Therefore, investigators have suggested that MBM miRNAs affect infant health and development $(5,9,10)$. This idea is supported by a study in which arthritic mice who fed milk rich in immune-related miRNAs (miR-30a, miR-223, and miR-92a) exhibited a reduction in cartilage depletion and inflammation (14).

Although many components of pMBM have been described, no study has examined how pMBM differs from tMBM in its composition of miRNA. When considering nutrient composition differences of $\mathrm{pMBM}$ and the proposed importance of miRNAs on infant health, a functional difference in miRNA content seems likely. This study tests the hypothesis that miRNA profiles differ between pMBM and tMBM samples collected 1 month post delivery, and that miRNAs "altered" in pMBM will have functional relevance to infant metabolism and immune regulation.

\section{METHODS}

This study was approved by the Institutional Review Board at the Penn State College of Medicine. All participants provided informed consent before participation.

\section{Term Milk Recruitment and Collection}

tMBM was collected as part of a follow-up study to the Intervention Nurses Start Infants Growing on Healthy Trajectories (INSIGHT) investigation (15), which examined the influence of genetics and environment on the growth and development of second-born siblings. This cohort included multiparous mothers $\geqslant 20$ years of age who had given birth to singleton, full-term infants $(\geqslant 38$ weeks gestation). Exclusion criteria for this group included medical complications with delivery and prenatal ultrasound, suggesting intrauterine growth retardation or newborn birth weight $<2,500 \mathrm{~g}$. Basic health and demographic information was obtained for each mother-infant dyad, including maternal age (years), pre-pregnancy weight $(\mathrm{kg})$, pregnancy weight gain $(\mathrm{kg})$, basic metabolic index $\left(\mathrm{kg} / \mathrm{m}^{2}\right)$ at delivery, maternal race/ethnicity, presence or absence of maternal hypertension, delivery method (cesarean section or vaginal delivery), infant gestational age (weeks), and infant sex (Table 1). The 36 tMBM samples utilized from INSIGHT study participants were chosen at random, and researchers were blinded to the maternal-infant characteristics. The medical and demographic characteristics of the present cohort are reflective of those present in the larger INSIGHT investigation (15). Term MBM samples were collected during the first $48 \mathrm{~h}$ after delivery (tColostrum; $n=13$ ) and 3-4 weeks after delivery (tMBM) - both before feeding (foremilk; $n=10$ ) and after feeding (hindmilk; $n=13$ ). No study has yet identified differences in the miRNA content of foremilk and hindmilk MBM, and none were identified in our subset of samples (Supplementary Table S8 online). Therefore, foremilk and hindmilk samples were combined as part of the tMBM analysis. MBM samples were manually expressed by participants into $50 \mathrm{ml}$ RNAse-free conical collection tubes (ThermoFisher Scientific, Waltham, MA, USA) after self-cleaning the nipple with soap and water. Samples were stored at $-4{ }^{\circ} \mathrm{C}$ for up to $24 \mathrm{~h}$ before collection by research staff and storage at $-80^{\circ} \mathrm{C}$ while awaiting analysis.

\section{Preterm Milk Recruitment and Collection}

pMBM was collected as a part of an ongoing larger study to identify relationships between genetic variation, diet, and gastrointestinal microbiota in preterm infants. This cohort includes both primiparous and multiparous women, 18-40 years of age giving birth to premature infants (28-37 weeks gestation) that require care in the Neonatal Intensive Care Unit. All breast-feeding mothers involved in the initial study were asked to provide an MBM sample as a part of the ongoing microbiota investigation. A subset of those samples was chosen at random for inclusion in the current study by researchers blinded to maternal-infant characteristics. Mother-infant dyads were not excluded from the pMBM cohort based on birth weight or medical complications such as emergent Cesarean delivery. The pMBM samples $(n=31)$ were all collected 3-4 weeks after delivery by expressing milk from both breasts using an electric Medela Lactina or Symphony breast pump (McHenry, IL, USA). No distinction was made between fore- and hindmilk collection in this cohort. A $10 \mathrm{ml}$ aliquot of $\mathrm{MBM}$ was stored in a sterile container at $4{ }^{\circ} \mathrm{C}$ for up to $24 \mathrm{~h}$ and then frozen at $-80^{\circ} \mathrm{C}$ until samples could be retrieved for analysis. Basic health and demographic information was obtained for each mother-infant dyad, including maternal age, maternal race/ethnicity, presence of maternal hypertension, delivery method (cesarean section or vaginal), infant gestational age (weeks), and infant sex (Table 1).

\section{RNA Extraction}

RNA was extracted with a Norgen Circulating and Exosomal RNA Purification Kit (Norgen Biotek, Ontario, Canada) as per manufacturer instructions. Samples were flash-thawed and spun for $20 \mathrm{~min}$ at $4{ }^{\circ} \mathrm{C}$ at $800 \mathrm{rpm}$ to separate the lipid and skim fractions. For each sample, $50 \mu \mathrm{l}$ of lipid and $200 \mu \mathrm{l}$ of skim milk were pipeted into separate $50 \mathrm{ml}$ tubes. When there was not a sufficient amount of lipid or skim, the maximum amount that could be extracted was used. All samples were diluted to $1 \mathrm{ml}$ using PBS. A two-step elution procedure was employed using $50 \mu \mathrm{l}$ of Elution solution centrifuged at 2,000 rpm for $2 \mathrm{~min}$ and then $3 \mathrm{~min}$ at $14,000 \mathrm{rpm}$. This was followed by a second elution using the $50 \mu \mathrm{l}$ of initial flowthrough centrifuged for only $3 \mathrm{~min}$ at 14,000 rpm. The final RNA concentration was determined on a Nanodrop Spectrophotmeter (ThermoFisher Scientific, Waltham, MA, USA), and the extracted RNA was stored at $-80^{\circ} \mathrm{C}$ before sequencing.

\section{RNA Sequencing and Alignment}

Yield and quality of the RNA samples was assessed using an Agilent 2100 Bioanalyzer prior to library construction with the NEXTflex Small RNA-Seq Kit v3 (Bioo Scientific; Austin, Texas). Multiplexed samples were run on an Illumina HiSeq 2500 Instrument (San Diego, CA, USA) at a targeted depth of one million reads per sample. FastQ outputs were clipped, trimmed, and filtered to a maximum read length of 30 base pairs using the FASTX Toolkit Module (Cold Spring Harbor, NY, USA) in Mobaxterm (Toulouse, France). Reads were aligned to the hg38 build of the human genome in Partek Flow (Partek; St Louis, Missouri) using the Bowtie 2 algorithm. Total miRNA counts within each sample were quantified with miRBase 
mature microRNAs v21, and read counts were normalized across samples using a trimmed mean of M-values (TMM) method. Only miRNAs with raw read counts greater than five in at least $50 \%$ of samples were evaluated in the differential expression analysis. A multi-model approach employing gene set analysis (GSA) in Partek Flow was used to identify individual miRNAs with differential expression between tMBM and pMBM samples collected 3-4 weeks post delivery (in lipid and skim fractions, respectively). Differential expression was defined as a Benjamini Hochberg FDR $<0.05$. The data set supporting the results of this article will be made available in the NCBI Sequence Read Archive following acceptance for publication.

\section{Functional Annotation}

The miRNAs with differential expression across tMBM and $\mathrm{pMBM}$ fractions underwent functional annotation analysis in DIANA mirPath v3 online software (http://snf-515788.vm.okeanos.grnet.gr/). The microT-CDS algorithm was used to identify mRNA targets for each miRNA of interest. MicroT-CDS uses miRBase v18 and Ensembl v69 for computational prediction of interactions between miRNAs and protein-coding genes in a species-specific manner (16). DIANA mirPath identified the KEGG pathways and GO categories with significant enrichment (FDR <0.05) for the miRNA targets in each MBM fraction. A list of high-confidence mRNA targets from each MBM fraction (microT-CDS score >0.98) was interrogated for protein-protein interaction networks using moderate stringency settings (interaction score $>0.40$ ) in the String v10 software (http:// string-db.org). String employs BIP, BioGRID, HPRD, IntAct, MINT, and PDP databases to identify biochemical interactions between lists of mRNA-protein products (17). Biological pathways overrepresented in high-confidence target gene sets were reported. Finally, for the lipid fraction of pMBM (which showed functional enrichment for metabolic processes), a list of 22 genes highly implicated in human obesity (18) were interrogated for target overlap with pMBM miRNAs of interest using miRDB software (http://www.mirdb.org).

\section{Statistical Analysis}

Analysis of maternal/infant medical and demographic data with a two-tailed student's $t$-test was performed to identify differences between term and preterm groups. A power analysis determined the sample size necessary for differentiating miRNAs among pMBM and tMBM cohorts. Assuming a logarithmic distribution of miRNA expression, it was determined that $22 \mathrm{MBM}$ samples per group provided $80 \%$ power to detect 1.8 -fold differences among 500 miRNAs (per-gene alpha $=0.002$ ). On the basis of these a priori calculations, we collected $23 \mathrm{tMBM}$ and $31 \mathrm{pMBM}$ samples and examined differences across 465 miRNAs in lipid MBM fractions. Individual miRNAs with significant differences between tMBM and pMBM fractions were identified using GSA analysis with Benjamini Hochberg FDR correction. Those miRNAs with FDR $<0.05$ were considered to have significant changes between groups. Fold change in miRNA levels between groups was reported as $\log 2$ values. The mRNA targets and their biological pathways were analyzed for functional enrichment with Fisher's exact tests, using a $2 \times 2$ table to compare the ratio of identified mRNA targets to the number of targets expected by chance. A nonparametric Kruskal-Wallis test was used to examine differences in individual miRNAs across lipid fractions of tMBM, pMBM, and tColostrum. Low volumes of colostrum produced following delivery do not allow for separation of skim and lipid fractions. Thus, tColostrum samples were compared only with lipid fractions of $\mathrm{tMBM}$ and $\mathrm{pMBM}$ (given their similar high-fat content). This comparison provided insight into how MBM miRNA might evolve following delivery (tColostrum vs. tMBM at 1 month) while also investigating the influence of maternal and infant characteristics on MBM miRNA profiles. Spatial relationships between total miRNA profiles for each group were examined with a two-dimensional PLS-DA using the MetaboAnalyst software (McGill; Montreal, Quebec, Canada). Hierarchical clustering was performed for the three groups using a Pearson distance measure of the top 20 miRNAs on Kruskal-Wallis testing with a complete clustering algorithm. Finally, the relationship between maternal/ infant medical and demographic characteristics and MBM miRNAs of interest were examined with Pearson correlation coefficients.

\section{RESULTS}

\section{Medical and Demographic Characteristics}

There was no significant difference in maternal age $(P=0.2)$, maternal race/ethnicity (\% white; $P=0.4$ ), or infant sex $(P=0.6)$ between the pMBM and tMBM groups. Within the pMBM group, there was a higher percentage of mothers with gestational hypertension $(P=0.0008)$ and a higher percentage of cesarean deliveries $(P=0.008)$. Gestational age at the time of delivery was significantly lower in the $\mathrm{pMBM}$ group $(P=4 \mathrm{E}-19)$ (Table 1$)$.

\section{Comparison of tMBM and pMBM Lipid Fractions}

Of the 2,588 mature human miRNAs interrogated for expression levels in $\mathrm{pMBM}$ and $\mathrm{tMBM}, 465$ were measured in the lipid fractions (Supplementary Table S1). There were 113 miRNAs with significant expression differences (false discovery rate $(F D R)<0.05)$ between tMBM and pMBM lipid samples. Sixty-eight of the miRNAs were downregulated in pMBM lipid fractions and 45 were upregulated. Eighty-two of these miRNAs have been described in previous studies of MBM $(6,14)$. The 15 miRNAs with the most significant differences across tMBM and pMBM lipid fractions (Table 2) underwent functional analysis. Of these, two pairs of miRNAs have overlapping seed sequences (miR-1260a/miR-1260b and miR378a-3p/miR378c) and six miRNAs have been previously described in breast tissue, breast milk, or breast discharge (19-24).

The 15 miRNAs most altered in the lipid fraction of pMBM had a total of 4,401 predicted mRNA targets in DIANA mirPath v3. These mRNA targets were associated with 90 gene ontology (GO) categories and 24 KEGG pathways (Supplementary Table S2). Notable GO categories with targeted enrichment included cellular nitrogen compound metabolic process, biosynthetic process, catabolic process, symbiosis, and viral process. Filtering down to the top 247 high-confidence mRNA targets with target scores $\geq 0.980$ revealed 15 mRNAs targeted by more than one miRNA on the list. Analysis of the 247 mRNAs in String v10 revealed a significant protein-protein interaction network $(P=8.1 \mathrm{E}-4)$ containing 230 nodes and 148 edges with a clustering coefficient of 0.752 (Supplementary Figure S1). Analysis of this network identified 13 biologic processes with enrichment (Table 3). Of note, three of the top five GO processes were involved in negative regulation of cellular macromolecule biosynthesis and each included more than 30 genes. In addition, an investigation of 22 unique genes implicated in human obesity (18) revealed that 19 genes had predicted miRNA interactions, and 13 of these were targeted by a miRNA of interest in lipid pMBM. For two obesity-related genes (adrenergic, beta-receptor 3, ARDB3; and Nuclear receptor sub-family 3 , group $\mathrm{C}$, member $1, \mathrm{NR} 3 \mathrm{C} 1$ ), there was 
Table 2. The 15 miRNAs most "altered" in the lipid fraction of pMBM relative to lipid tMBM one month post delivery

\begin{tabular}{|c|c|c|c|c|c|c|}
\hline $\begin{array}{l}\text { Top } 15 \text { miRNAs } \\
\text { (lipid fraction) }\end{array}$ & FDR & $\begin{array}{l}\text { Fold change } \\
\text { (pMBM) }\end{array}$ & $\begin{array}{l}\text { Seed } \\
\text { sequence }\end{array}$ & $\begin{array}{l}\text { Overlapping miRNA } \\
\text { seeds }\end{array}$ & $\begin{array}{l}\text { Target mRNAs (total/ } \\
\text { high-confidence) }\end{array}$ & $\begin{array}{l}\text { Breast-related } \\
\text { expression }\end{array}$ \\
\hline hsa-miR-4470 & $4.31 E-06$ & $6.75 E+01$ & GGCAAAC & None & $633 / 63$ & \\
\hline hsa-miR-4687-3p & $4.31 E-06$ & $-3.54 \mathrm{E}+00$ & GGCUGUU & None & $428 / 46$ & $\begin{array}{l}\text { Nipple discharge from } \\
\text { intraductal papilloma }^{a}\end{array}$ \\
\hline hsa-miR-1260a & $9.25 E-06$ & $-2.10 \mathrm{E}+00$ & UCCCACC & hsa-miR-1260b & 39013 & \\
\hline hsa-miR-1260b & $1.70 E-05$ & $-2.01 E+00$ & UCCCACC & hsa-miR-1260a & $73 / 2$ & $\begin{array}{l}\text { MCF-7 breast cancer } \\
\text { cells }^{b}\end{array}$ \\
\hline hsa-miR378a-3p & $1.70 E-05$ & $-1.97 E+00$ & CUGGACU & $\begin{array}{l}\text { hsa-miR-378b/c/d/e/f/h/ } \\
\text { I hsa-miR-422a }\end{array}$ & $231 / 9$ & $\begin{array}{l}\text { Breast milk, MCF-7 } \\
\text { breast cancer cells }{ }^{c}\end{array}$ \\
\hline hsa-miR-378g & $3.35 E-05$ & $-2.10 \mathrm{E}+00$ & CUGGGCU & None & $405 / 16$ & \\
\hline hsa-miR-378c & $5.36 E-05$ & $-1.88 \mathrm{E}+00$ & CUGGACU & $\begin{array}{l}\text { hsa-miR378a/b/d/e/f/h/l } \\
\text { hsa-miR-422a }\end{array}$ & $255 / 12$ & \\
\hline hsa-miR-4474-5p & $5.94 \mathrm{E}-05$ & $3.33 E+01$ & UAGUCUC & None & $227 / 14$ & $\begin{array}{l}\text { Plasma in unexplained } \\
\text { recurrent spontaneous } \\
\text { abortion }^{d}\end{array}$ \\
\hline hsa-miR-6763-3p & $5.94 \mathrm{E}-05$ & $-2.19 E+00$ & UCCCCGG & None & $17 / 1$ & \\
\hline hsa-miR-5585-5p & $7.16 E-05$ & $-1.70 E+00$ & GAAGUAC & None & $342 / 18$ & \\
\hline hsa-miR-1200 & $3.39 E-04$ & $-1.64 \mathrm{E}+00$ & UCCUGAG & None & $597 / 26$ & $\begin{array}{l}\text { MCF-7 breast cancer } \\
\text { cells }^{\mathrm{e}}\end{array}$ \\
\hline hsa-miR-4783-5p & $3.44 E-04$ & $-1.99 E+00$ & GCGCGCC & None & $0 / 0$ & \\
\hline hsa-miR-6772-3p & $4.39 E-04$ & $4.97 E+00$ & UGCUCCU & None & $88 / 7$ & \\
\hline hsa-miR-187-5p & $5.25 E-04$ & $-1.78 \mathrm{E}+00$ & GCUACAA & None & $173 / 9$ & $\begin{array}{l}\text { BRCA1 triple negative } \\
\text { breast cancer cells }{ }^{f}\end{array}$ \\
\hline hsa-miR-6510-5p & $5.25 E-04$ & $-1.99 E+00$ & AGCAGGG & None & $542 / 17$ & \\
\hline $\begin{array}{l}\text { pMBM, premature ma } \\
\text { a Zhang et al. (24). } \\
{ }^{\mathrm{b} G o n u l ~ e t ~ a l . ~(20) . ~} \\
{ }^{\mathrm{C}} \text { lkeda et al. (19). } \\
{ }^{\mathrm{d}} \text { Qin et al. (21). } \\
{ }^{\mathrm{e}} \text { Shah et al. (22). } \\
{ }^{\mathrm{f}} \text { Matamala et al. (23). } \\
\text { Target mRNAs identif }\end{array}$ & al breast milk & $=31) ; \mathrm{tMBM}$, term & naternal breast & $n=23)$ & 098 & \\
\hline
\end{tabular}

consistent upregulation of all target miRNAs present in lipid pMBM. For three other obesity-related genes (Leptin, LEP; tumor necrosis factor, TNF; and uncoupling protein 3, UCP3), there was consistent downregulation of all target miRNAs present in lipid pMBM.

\section{Comparison of tMBM and pMBM Skim Milk Fractions}

There were fewer miRNAs identified in skim milk fractions. Thirty-two miRNAs showed robust expression in the skim milk fractions of both tMBM and pMBM (Supplementary Table S3). All 32 of the miRNAs measured in skim milk fractions were also found in lipid fractions. In the skim fractions, there were 12 miRNAs with significant expression differences between pMBM and tMBM samples (Table 4). Seven of the 12 miRNAs were downregulated in skim fractions of $\mathrm{pMBM}$ and five were upregulated. Three of these miRNAs have been described in previous studies of MBM and nine are novel $(6,9,10,14)$. Of these 12 miRNAs, two pairs have overlapping seed sequences (miR-1260a/ $\mathrm{miR}-1260 \mathrm{~b}$ and $\mathrm{miR} 378 \mathrm{a}-3 \mathrm{p} / \mathrm{miR} 378 \mathrm{c}$ ), and five have been previously described in breast tissue or breast milk $(19,20,25-27)$.

The 12 miRNAs with significant differences in expression in pMBM had a total of 2,767 predicted mRNA targets in DIANA mirPath v3. These mRNAs showed enrichment for $10 \mathrm{GO}$ categories and 9 KEGG pathways (Supplementary Table S4). The most significantly enriched GO class was molecules involved in nitrogen metabolism. The top three KEGG pathways included genes involved in glycosphingolipid biosynthesis, lysine degradation, and calcium signaling. There were 124 mRNA targets that survived the high-confidence filter, and 10 of these were targeted by more than one mRiNA on the list. Functional analysis of the 124 mRNAs in String v10 demonstrated a significant protein-protein interaction network $(P=0.034)$ containing 111 nodes and 23 edges with a clustering coefficient of 0.892 (Supplementary Figure S2); however, GO analysis of this network failed to identify a protein-protein interaction pathway with significant enrichment. 
Table 3. The miRNAs differentially expressed in lipid pMBM target gene ontology (GO) pathways related to macromolecule and cellular biosynthetic metabolism

\section{Pathway ID Pathway description \\ G0.0045892 Negative regulation of transcription, DNA-templated}

GO.2000113 Negative regulation of cellular macromolecule biosynthetic process

GO.0010629 Negative regulation of gene expression

GO.0010558 Negative regulation of macromolecule biosynthetic proces

G0.0031327 Negative regulation of cellular biosynthetic process

G0.0045934 Negative regulation of nucleobase-containing compound metabolic process

G0.0050794 Regulation of cellular process

Gene count

31

0.0046

\section{Target mRNAs with protein products in network}

ARID5A, BMI1, CEBPG, CTBP1, CYP1B1, EGLN1, EHMT1, FBXW11, FOXF2, FOXG1, H3F3B, IKZF4, JAZF1, MECOM, MKX, NAB1, NR2F2, PAX5, PHF21A, POU2F1, TDG, TFAP2A, TFCP2L1, TSG101, UBE2D2, UBE2D3, WWC2, ZEB2, ZFHX3, ZNF217, ZNF675

34

0.0046 ARID5A, BMI1, CEBPG, CELF1, CTBP1, CYP1B1, EGLN1, EHMT1, FBXW11, FBXW7, FOXF2, FOXG1, H3F3B, IKZF4, JAZF1, MECOM, METTL3, MKX, NAB1, NR2F2, PAX5, PHF21A, POU2F1, TDG, TFAP2A, TFCP2L1, TSG101, UBE2D2, UBE2D3, WWC2, ZEB2, ZFHX3, ZNF217, ZNF675

35

0.0069 ARID5A, BMI1, CEBPG, CELF1, CTBP1, CYP1B1, DYRK1A, EGLN1, EHMT1, FBXW11, FLOT2, FOXF2, FOXG1, H3F3B, IKZF4, JAZF1, MECOM, METTL3, MKX, NAB1, NR2F2, PAX5, PHF21A, POU2F1, TDG, TFAP2A, TFCP2L1, TSG101, UBE2D2, UBE2D3, WWC2, ZEB2, ZFHX3, ZNF217, ZNF675

0.0103 ARID5A, BMI1, CEBPG, CELF1, CTBP1, CYP1B1, EGLN1, EHMT1, FBXW11, FBXW7, FOXF2, FOXG1, H3F3B, IKZF4, JAZF1, MECOM, METTL3, MKX, NAB1, NR2F2, PAX5, PHF21A, POU2F1, TDG, TFAP2A, UBE2D2, UBE2D3, WWC2, ZEB2, ZFHX3, ZNF217, ZNF675

0.0103 ADRA2C, ARID5A, BMI1, CEBPG, CELF1, CTBP1, CYP1B1, EGLN1, EHMT1, FBXW11, FBXW7, FOXF2, FOXG1, H3F3B, IKZF4, JAZF1, MECOM, METTL3, MKX, NAB1, NR2F2, PAX5, PHF21A, POU2F1, TDG, TFAP2A, UBE2D2, UBE2D3, WWC2, ZEB2, ZFHX3, ZNF217, ZNF675

0.0103 ADRA2C, ARID5A, BMI1, CEBPG, CTBP1, CYP1B1, DYRK1A, EGLN1, EHMT1, FBXW11, FBXW7, FOXF2, FOXG1, H3F3B, IKZF4, JAZF1, MECOM, MKX, NAB1, NR2F2, PAX5, PHF21A, POU2F1, TDG, TFAP2A, UBE2D2, UBE2D3, WWC2, ZEB2, ZFHX3, ZNF217, ZNF675

0.0103 ABI2, ADCYAP1, ADRA2C, ALMS1, ATAD2, ATF6B, AZIN1, BCL2L2, BMI1, BMPR1A, C15orf23, CACNA2D4, CAMSAP2, CCNY, CD47, CDC42BPG, CEBPG, CELF1, CENPB, COBL, COLQ, CPLX1, CTBP1, CYP1B1, DACT2, DSCAM, DYRK1A, EGLN1, EHMT1, EIF3D, ELL2, ESPL1, ETV1, FBXW7, FLOT2, FNDC5, FOXO1, GCC2, GDI1, GLS2, GPR110, H3F3B, HIPK3, HNRNPH1, IL36RN, ITSN2, JAZF1, KCNA4, KDM6A, FOXO1, GCC2, GDI1, GLS2, GPR110, H3F3B, HIPK3, HNRNPH1, IL36RN, ITSN2, JAZF1, KCNA4, KDM6A,
KHDRBS2, KPNA1, LECT1, LTF, MAP1A, MAP3K12, METTL3, MKX, MYO18A, MYO1C, MYRIP, NAB1, NAMPT, NR2F2, NREP, OSTF1, PABPN1, PAX5, PDE10A, PDE7A, PHACTR4, PHF21A, PITPNC1, PKD1, PLCZ1, POLB, POU2F1, PPM1L, PPP1R3F, PPP3CB, PRKAR2A, PTPRG, PTX3, PXDN, RAB3B, RAB6C, RABL3, RALBP1, RC3H2, RFWD2, RIPK2, RPAP2, SEC62, SEMA3A, SESN3, SLC2A8, SLC30A1, SLITRK5, SNCAIP, SNX27, SRF, STK4, SYNJ1, TACC1, TDG, TEX15, TFAP2A, TIRAP3, TMEM64, TMEM67, TMX2, UBAC2, UBN2, UNC13C, USP31, WWC2, YY1AP1, ZBTB44, ZEB2, ZNF217, ZNF286B, ZNF451, ZNF675

G0.0051172 Negative regulation of nitrogen compound metabolic process

GO.0051253 Negative regulation of RNA metabolic process

G0.1903507 Negative regulation of nucleic acid-templated transcription

G0.0050789 Regulation of biological process
0.0103 ADRA2C ARID5A, BMI1, CEBPG, CELF1, CTBP1, CYP1B1, DYRK1A, EGLN1, EHMT1, FBXW11, FBXW7, FOXF2, FOXG1, H3F3B, IKZF4, JAZF1, MECOM, METTL3, MKX, NAB1, NR2F2, PAX5, PHF21A, POU2F1, TDG, TFAP2A, UBE2D2, UBE2D3, WWC2, ZEB2, ZFHX3, ZNF217, ZNF675

300.0103 ARID5A, BMI1, CEBPG, CTBP1, CYP1B1, DYRK1A, EGLN1, EHMT1, FBXW11, FOXF2, FOXG1, H3F3B, IKZF4, JAZF1, MECOM, MKX, NAB1, NR2F2, PAX5, PHF21A, POU2F1, TDG, TFAP2A, UBE2D2, UBE2D3, WWC2, ZEB2, ZFHX3, ZNF217, ZNF675

0.0103 ARID5A, BMI1, CEBPG, CTBP1, CYP1B1, EGLN1, EHMT1, FBXW11, FOXF2, FOXG1, H3F3B, IKZF4, JAZF1, MECOM, MKX, NAB1, NR2F2, PAX5, PHF21A, POU2F1, TDG, TFAP2A, UBE2D2, UBE2D3, WWC2, ZEB2, ZFHX3, ZNF217, ZNF675
0.0181 ABI2, ADCYAP1, ADRA2C, ALMS1, APC, ATAD2, ATF6B, ATG12, AZIN1, BCL2L2, BMI1, BMPR1A, C15orf23, CACNA2D4, CAMSAP2, CCNY, CD47, CDC42BPG, CEBPG, CELF2, CENPB, COBL, COLQ, CPLX1, CTBP1, CYP1B1, DACT2, DBF4, DSCAM, DYRK1A, EGLN1, EHMT1, EIF3D, ELL2, ENPEP, ESPL1, ETV1, FBXW7, FLOT2, FNDC5, FOXO1, GCC2, GCH1, GDI1, GLS2, GPR110, H3F3B, HIPK3, HNRNPH1, ITSN2, JAZF1, KCNA4, KDM6A, KHDRBS2, KPNA1, LIPC, LTF, MAP1A, MAP3K12, METTL3, MKX, MYO18A, MYO1C, MYRIP, NAMPT, NR2F2, NREP, OSTF1, PABPN1, PAX5, PDE10A, PDE7A, PHACTR4, PHF21A, PITPNC1, PKD1, PLCZ1, POLB, POU2F1, PPM1L, PPP1R3F, PPP3CB, PRKAR2A, PTPRG, PTX3, 
Combined analysis of pMBM Skim and Lipid Fractions

When comparing both lipid and skim fractions of pMBM with $\mathrm{tMBM}$ fractions, we identified nine differentially expressed miRNAs (miR378a-3p, miR378c, miR-378g, miR-1260a, miR-1260b, miR-4783-5p, miR-4784, miR-5787, and miR-7975). All nine miRNAs were altered in the same direction in skim and lipid fractions of pMBM with the exception of miR-4783-5p (which was upregulated in the skim pMBM fraction but downregulated in the lipid pMBM fraction). Two miRNAs were upregulated in both fractions of pMBM (miR-5787 and miR-4784) and six were downregulated. Functional analysis of the nine miRNAs with differential expression patterns in both the lipid and skim fractions of pMBM yielded 2,614 predicted mRNA targets in DIANA mirPATH v3. These mRNAs showed enrichment for 42 GO categories and 5 KEGG pathways (Supplementary Table S5). Top GO and KEGG targets included networks of mRNAs related to micronutrient metabolism, such as biosynthetic processes, cellular nitrogen metabolism, glycosphingolipid biosynthesis, and lysine degradation. Eight of the nine miRNAs also targeted 62 genes involved in viral process.

\section{Comparison of pMBM with tMBM and tColostrum}

A Kruskal-Wallis test was used to examine differences in individual miRNAs between lipid tMBM (collected 3-4 weeks post delivery), lipid pMBM (collected 3-4 weeks post delivery), and tColostrum samples (collected within $48 \mathrm{~h}$ of delivery). There were 127 miRNAs with significant differences among these groups (Supplementary Table S6). For most of the miRNAs interrogated, expression patterns in tMBM and tColostrum trended together in the opposite direction of pMBM (Supplementary Figure S3). Partial least squares discriminant analysis (PLS-DA) analysis achieved partial separation of the three sample types based on total miRNA profile (Figure 1). A hierarchical clustering analysis using the 20 miRNAs with the most significant changes across the three groups demonstrated complete separation of pMBM samples from both $\mathrm{tMBM}$ and tColostrum samples (Figure 2). Note that duplicate (foremilk and hindmilk) tMBM samples (e.g., tMBM 1a and tMBM 1b) tended to cluster together. In addition, tColostrum and $\mathrm{tMBM}$ samples from the same mother (e.g., tColostrum 7 and tMBM 7a) often cluster near one another.

\section{Relationship of Medical/Demographic Characteristics and MBM miRNA}

A Pearson correlation analysis was used to explore the relationships between medical/demographic variables and miRNA expression. This analysis identified significant correlations between gestational age (measured as a continuous variable) and 21 of the 26 miRNAs "altered" in lipid or skim fractions of pMBM samples (Supplementary Table S7). Six of the 26 miRNAs were correlated with method of delivery, but none correlated with maternal race/ethnicity, presence of maternal hypertension, twin gestation, or infant 


\section{Articles | Carney et al.}

Table 4. The 12 miRNAs "altered" in the skim fraction of pMBM relative to skim tMBM one month post delivery

\begin{tabular}{|c|c|c|c|c|c|c|}
\hline $\begin{array}{l}\text { Top } 12 \text { miRNAs } \\
\text { (skim fraction) }\end{array}$ & FDR & $\begin{array}{l}\text { Fold change } \\
\text { (pMBM) }\end{array}$ & Seed sequence & $\begin{array}{l}\text { Overlapping miRNA } \\
\text { seeds }\end{array}$ & $\begin{array}{l}\text { Target mRNAs (total/ } \\
\text { high-confidence) }\end{array}$ & Breast-related expression \\
\hline hsa-miR-378g & $1.58 \mathrm{E}-04$ & $-2.12 \mathrm{E}+00$ & CUGGGCU & & $405 / 16$ & \\
\hline hsa-miR378a-3p & $6.40 E-04$ & $-1.90 \mathrm{E}+00$ & CUGGACU & $\begin{array}{l}\text { hsa-miR-378b/c/d/e/f/h/l } \\
\text { hsa-miR-422a }\end{array}$ & $231 / 9$ & $\begin{array}{l}\text { Breast milk, MCF-7 breast } \\
\text { cancer cells }{ }^{a}\end{array}$ \\
\hline hsa-miR-5787 & $6.40 E-04$ & $2.50 \mathrm{E}+00$ & GGCUGGG & hsa-miR-4505 & $728 / 29$ & \\
\hline hsa-miR-1260a & $7.47 \mathrm{E}-04$ & $-1.77 E+00$ & UCCCACC & hsa-miR-1260b & $390 / 13$ & \\
\hline hsa-miR-1260b & $7.84 \mathrm{E}-04$ & $-1.66 \mathrm{E}+00$ & UCCCACC & hsa-miR-1260a & $73 / 2$ & MCF-7 breast cancer cells ${ }^{b}$ \\
\hline hsa-miR-378c & $7.84 \mathrm{E}-04$ & $-1.69 E+00$ & CUGGACU & $\begin{array}{l}\text { hsa-miR-378b/c/d/e/f/h/l } \\
\text { hsa-miR-422a }\end{array}$ & $255 / 12$ & \\
\hline hsa-miR-7975 & $7.84 \mathrm{E}-04$ & $-1.67 E+00$ & UCCUAGU & & $50 / 0$ & \\
\hline hsa-miR-7704 & $9.03 E-04$ & $2.12 \mathrm{E}+00$ & GGGGUCG & & $13 / 1$ & \\
\hline hsa-miR-4784 & $1.58 \mathrm{E}-02$ & $1.76 \mathrm{E}+00$ & GAGGAGA & hsa-miR-3150b-3p & $482 / 39$ & $\begin{array}{l}\text { BRCA1 triple negative breast } \\
\text { cancer cells }{ }^{c}\end{array}$ \\
\hline hsa-miR-4294 & $1.69 E-02$ & $1.69 E+00$ & GGAGUCU & & $114 / 1$ & Breast cancer cells ${ }^{d}$ \\
\hline hsa-miR-4783-3p & $3.11 \mathrm{E}-02$ & $2.24 \mathrm{E}+00$ & CCCGGUG & & $21 / 2$ & \\
\hline hsa-miR-4454 & $4.97 \mathrm{E}-02$ & $-1.39 E+00$ & GAUCCGA & & $5 / 0$ & Inflammatory breast cancer ${ }^{\mathrm{e}}$ \\
\hline \multicolumn{7}{|c|}{$\begin{array}{l}\text { pMBM, premature maternal breast milk }(n=31) \text {; tMBM, term maternal breast milk }(n=23) \text {. } \\
\text { Target mRNAs identified with DIANA miRPATH software. High-confidence targets included those with target score }>0.98 \text {. } \\
\text { alkeda et al. (19). } \\
{ }^{b} \text { Gonul et al. (20). } \\
{ }^{c} \text { Yang et al. (25). } \\
{ }^{d} \text { Kumari(26). }\end{array}$} \\
\hline
\end{tabular}

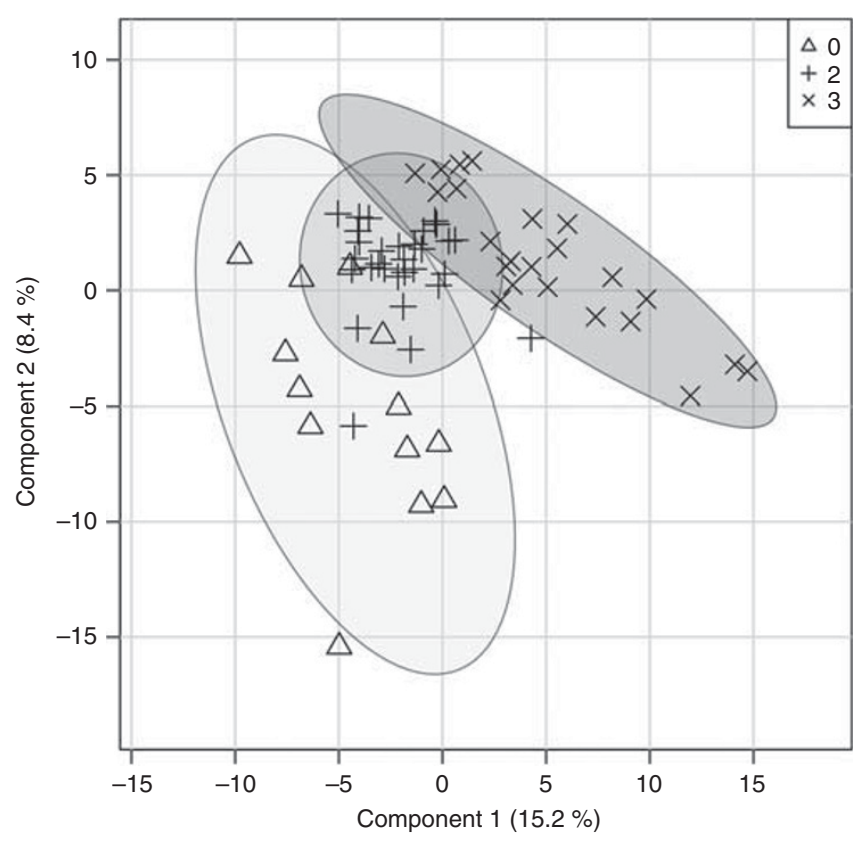

Figure 1. Partial least squares discriminant analysis. A partial least squares discriminant analysis (PLS-DA) of the total microRNA profile for pMBM (class 2,,$+ n=31$ ), tMBM (class 3, $x, n=23$ ), and tColostrum (class $0, \Delta, n=10$ ) achieved partial separation using two dimensions and accounted for $23.6 \%$ of the variance between samples.

sex. Of the six miRNAs correlated with delivery method, three were positively correlated, three were negatively correlated, and all six showed inverse patterns compared with gestational age. Heatmap visualization of Pearson's correlations demonstrated hierarchical clustering of gestational age with lipid and skim fractions of $\mathrm{miR}-1260 \mathrm{a} / \mathrm{b}$ as well as the lipid fraction of miR-6763-3p and the skim fraction of miR-7975 (Figure 3). All these factors were positively correlated.

\section{DISCUSSION}

The expression of miRNAs in the lipid and skim milk fractions of pMBM differs significantly from the expression in tMBM fractions 1 month after delivery. There are nine miRNAs "altered" across both fractions, and these miRNAs target a number of transcripts involved in metabolic processes. The pathway with the most significant enrichment in miRNA targets from pMBM is glycosphingolipid biosynthesis. Glycosphingolipids are important for neurodevelopment (28), are essential to a functioning cell membrane, and have a major role in signal transduction of lipid rafts (29). These same lipid domains may aid in the protected delivery of miRNAs to the infant gut, regulating gastrointestinal function, metabolism, and energy utilization.

\section{Potential Mechanisms for a Unique miRNA Profile in pMBM}

Differences in miRNA composition of pMBM and tMBM may result from regulation within the mammary epithelial cell nucleus (through pri-miRNA expression or pre-miRNA processing), or they may represent a response to environmental changes (such as abrupt premature delivery), which alters the way miRNAs are packaged and extruded into MBM. MiRNAs are packaged in a variety of ways, including vesicles, 


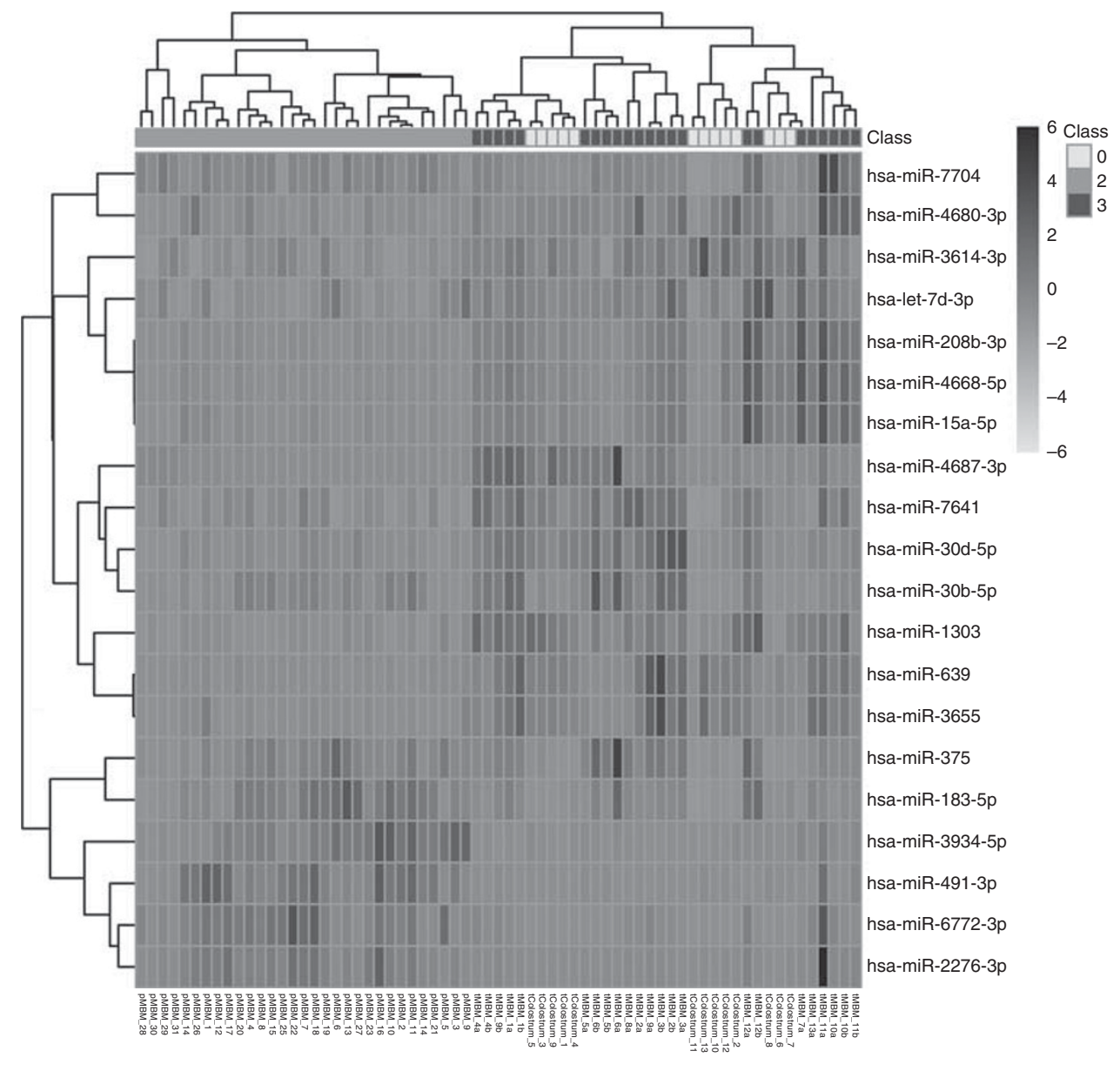

Figure 2. Hierarchical clustering $(\mathrm{HC})$ analysis. $\mathrm{HC}$ of the 20 miRNAs with the most significant changes across the three groups showed clustering of pMBM (class 2; $n=31$ ) from tMBM (class $3 ; n=23$ ) and tColostrum (class $0 ; n=13$ ). Note that tMBM foremilk $(n=10)$ and hindmilk $(n=13)$ samples (denoted a and b, respectively), as well as tMBM and tColostrum samples (taken from the same mother one month apart) were also spatially clustered. Gray-scale values indicate average $Z$-score of normalized abundance for each miRNA.

shedding, exosomal transfer, and RICS-complex proteinbinding (30). Because individual miRNAs show preference for specific packaging mechanisms (31), a change in the ratio of carriers (e.g., exosomes or nucleoproteins) could affect the secretion of certain miRNAs in pMBM. Given that tMBM and pMBM differ in macronutrient and micronutrient composition $(1,2)$, it is plausible that they might also differ in types of miRNA carriers.

Alternatively, premature delivery might influence production of miRNAs within the cell nucleus. Given the milieu of hormonal changes occurring pre- and post-partum, a shift in maternal hormones could influence miRNA transcription. For example, lactogenic hormones alter expression and secretion of miRNAs in cultured mammary cells (7). Mothers of preterm infants have lower prolactin levels (32), and this may alter expression and secretion of miRNAs. The miRNA profile in breast cells is altered by exposure to estrogen (33), and exposure to progesterone increases the levels of miRNA processing machinery, which could result in an altered miRNA profile (34). As estrogen and progesterone levels increase throughout pregnancy, mothers of premature babies have lower circulating levels of both hormones at delivery. This could have a lasting impact on the miRNAs in pMBM, providing potential evolutionary benefits for the premature neonate.

\section{Implications for Infant Health}

Premature infants face increased risk for a number of medical co-morbidities, including failure to thrive, sepsis, necrotizing enterocolitis (NEC), and neurodevelopmental delays. These risks may be potentiated or mitigated by maternal-infant transfer of MBM miRNAs. Notably, premature infants have a vastly different growth trajectory than term infants have, and metabolic pathways, such as the glycolytic pathway, are affected by miRNAs "altered" in pMBM (Table 3 and Supplementary Table S2). An investigation of miRNAs that target obesity-related genes (18) reveals changes relevant to growth and metabolism in the premature newborn. Interestingly, the miRNAs that target UCP3, LEP, and TNF are downregulated in lipid pMBM. UCP3 alters metabolism in response to fuel depletion, whereas TNF increases LEP production and adipocyte insulin resistance (35). Altogether, 


\section{Articles | Carney et al.}

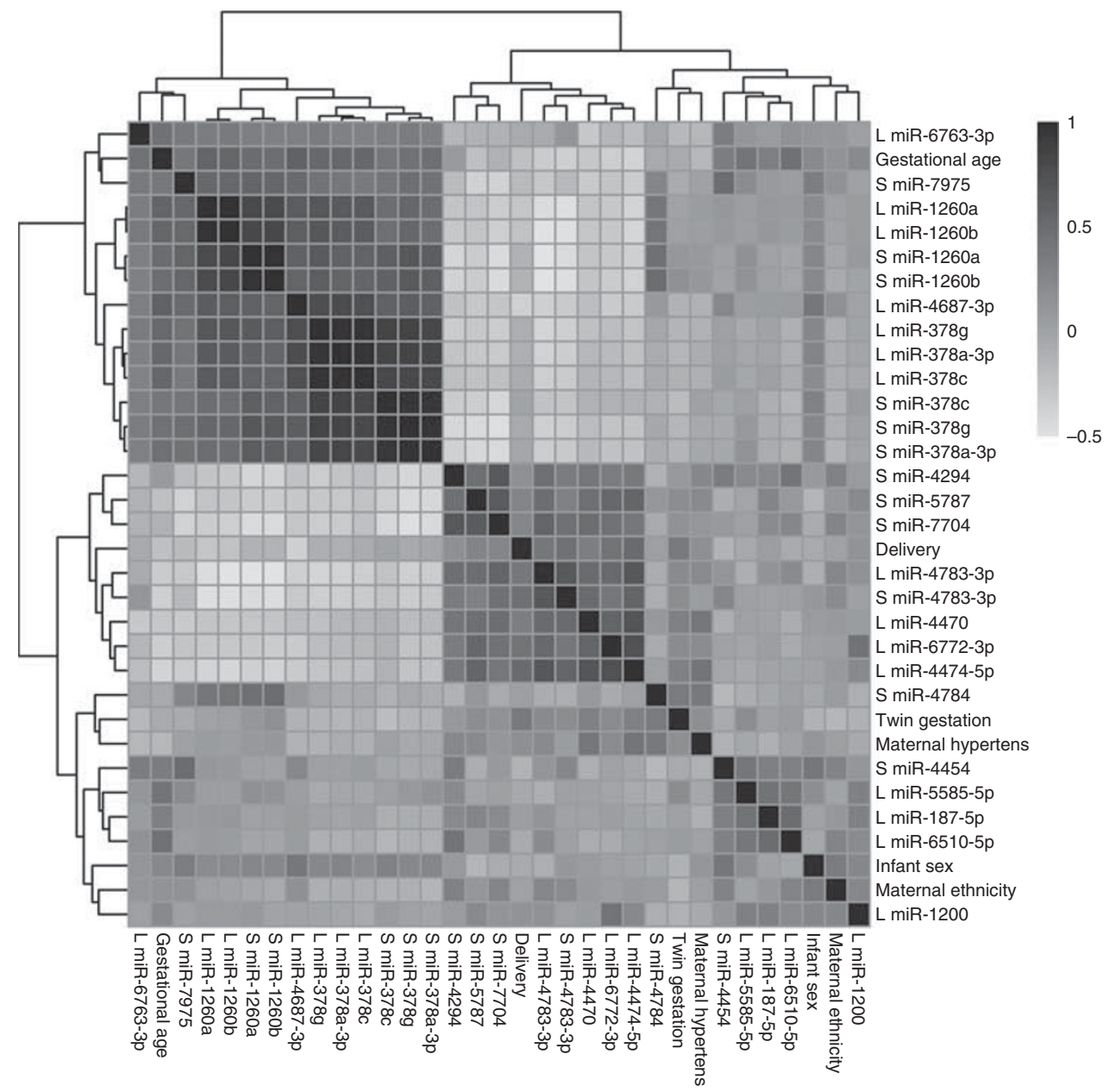

Figure 3. Pearson correlations. Heatmap visualization of Pearson's correlation for the 26 miRNAs altered in lipid or skim pMBM demonstrates correlations between gestational age (as a continuous variable) and 21 miRNAs. Six of these miRNAs were correlated with delivery method, but no correlation was found between miRNAs and other variables. Color represents Pearson $R$-values.

polymorphisms in UCP3 and TNF act synergistically to increase the risk for type 2 diabetes mellitus (36). Thus, widespread downregulation of miRNAs targeting these genes might influence glucose homeostasis in the premature infant. Conversely, upregulation of miRNAs in lipid pMBM that target ADRB3 and NR3C1 could lead to downregulation of their protein products in the premature infant. A decrease in ADRB3 and NR3C1, which are both negative regulators of adipogenesis $(37,38)$, could increase fat production and have functional benefits in the cachectic premature newborn.

Premature infants face increased risk of viral and bacterial infections. Previous studies have described enrichment of immune-related miRNAs in MBM $(5,10)$, and the current investigation identifies a pair of immune-related miRNAs (miR-1260a and miR-1260b) that are downregulated in pMBM compared with tMBM (Tables 2 and 4). Humanactivated B-cell lines demonstrate robust miR-1260 expression in comparison to indolent germinal center cells, suggesting that miR-1260 may influence B-cell proliferation (39). Thus, downregulation of miR-1260 in pMBM may not serve a protective function in premature infants, but it could contribute to their immune-susceptible state. Coupled with decreased miRNA-targeting of TNF in pMBM, these changes could have pathophysiologic implications in NEC. Conversely, robust expression of miR-1260a/miR-1260b in tMBM may impart immune protection on the infant gut and have a role in the decreased incidence of gastroenteritis in breast-fed infants (40).

\section{The Relative Influence of Prematurity on MBM miRNA}

To account for the vast differences between a 35-week and a 27-week preterm infant, the current study investigated relationships between MBM miRNA and gestational age as a continuous variable. This approach reaffirmed that 21 of the 26 miRNAs altered in pMBM vs. tMBM samples were significantly related to premature delivery. Furthermore, none of the 26 miRNAs influenced by gestational age were correlated with maternal race/ethnicity, maternal hypertension, twin gestation, or infant sex (Figure 3). Six of these miRNAs were correlated with cesarean section delivery, and 


\section{Premature birth and breast milk miRNA Articles}

this may represent an important health factor contributing to MBM miRNA shifts following premature delivery. These results suggest that maternal factors may influence MBM miRNA content. This is consistent with a previous study (13), showing that the expression of miRNAs in MBM was perturbed by a maternal high-fat diet. In fact, the miRNA composition of MBM may be influenced more by maternal factors than length of lactation (i.e., time since delivery). This idea is supported by Figure 2, showing that miRNA profiles for tMBM preferentially cluster alongside tColostrum from maternal pairs, rather than $\mathrm{PMBM}$ samples taken at the same duration post delivery. Premature delivery, acting as a health factor, may influence the miRNA profile of MBM for a substantial period of lactation. This has implications for infant growth trajectory throughout the breast-feeding period. It may explain why premature infants grow more appropriately on their mother's own milk compared with the donor milk from mothers delivering at term.

\section{Limitations}

The current study is limited by utilization of pMBM and tMBM samples collected from two separate investigations. This approach introduces differences in MBM collection methods (e.g., manual vs. machine-pump), which could influence miRNA content. MBM miRNA arises primarily from the mammary gland epithelium rather than maternal circulation (9), and the compressive nature of manual pumping could disrupt this epithelial layer, resulting in the collection of intracellular miRNA. To control for this, we have performed differential centrifugation to eliminate epithelial components in both $\mathrm{pMBM}$ and $\mathrm{tMBM}$ samples, focusing the analysis on extracellular miRNAs in lipid and skim milk layers. Furthermore, the miRNAs in the present analysis included only those miRNAs present in $>50 \%$ of samples. Thus, intracellular miRNAs introduced by a manual pumping technique should effectively be excluded from consideration.

An additional limitation of this study is its reliance on both foremilk and hindmilk samples within the tMBM cohort. Hindmilk, which is enriched in lipid content, but not lactose or protein, could demonstrate increased miRNA diversity through the stability of lipid-bound miRNAs. However, raw RNA read counts in hindmilk tMBM samples did not exceed those observed in foremilk samples. In addition, such differences should be eliminated by differential centrifugation and analysis of skim fractions, which removes lipid-bound miRNAs. One previous study examining differences in preand post-feed miRNAs within human milk cells identified 33 miRNAs with "alterations" post feed (11). Only three of these miRNAs (miR-1260b, miR-4454, and miR-671-3p) show differences in our comparison of pMBM and tMBM. This small overlap is likely related to removal of human milk cells by centrifugation. This technique focuses on MBM fractions with the highest likelihood for maternal-infant transfer.

A final limitation of the present investigation is its sample size. Previous studies have utilized 4-10 MBM samples for exploring miRNA profiles, but our a priori power analysis required a minimum of 22 samples per group. To power a comparison of tMBM and PMBM groups, we included 23 and 31 samples from these cohorts, respectively. Thus, our investigation is wellpowered for an analysis of 465 miRNAs across term and preterm cohorts, but is somewhat underpowered for comparison of tColostrum samples $(n=13)$, and comparisons between foremilk $(n=10)$ and hindmilk $(n=13)$ samples. Thus, these findings should be interpreted with caution.

\section{CONCLUSION}

This study has identified nine miRNAs with differences in lipid and skim fractions of pMBM compared with tMBM collected 3-4 weeks post delivery. Many of the genes targeted by these miRNAs influence metabolic pathways and could affect infant growth trajectory via maternal-fetal transfer. A subset of miRNAs was correlated with delivery method, and this may represent one medical factor that triggers changes in the MBM miRNA profile. These findings underscore how MBM, which is generally considered the optimum nutrition for infants, may be individually adapted to specific infant needs through the regulation of factors such as miRNA.

\section{SUPPLEMENTARY MATERIAL}

Supplementary material is linked to the online version of the paper at http://www.nature.com/pr

\section{ACKNOWLEDGMENTS}

We thank Jessica Beiler for assistance in sample collection, Frank Middleton for guidance in RNA analysis, and Yuka Imamura for expertise in RNA sequencing.

\section{STATEMENT OF FINANCIAL SUPPORT}

Funding provided by the Center for Research on Women and Newborn Health, The Gerber Foundation, the NIDDK (1R01DK099364-03), and the Departments of Surgery and Pediatrics at the Penn State College of Medicine.

Disclosure: Hicks holds a preliminary patent for microRNA biomarkers in autism spectrum disorder and serves as a consultant for Motion Intelligence. Kelleher serves as the president and CEO of LactoGenix. These conflicts of interest are unrelated to the current manuscript and are under an active management plan by the Penn State College of Medicine.

\section{REFERENCES}

1. Anderson GH, Atkinson SA, Bryan MH. Energy and macronutrient content of human milk during early lactation from mothers giving birth prematurely and at term. Am J Clin Nutr 1981;34:258-65.

2. Lemons JA, Moye L, Hall D, et al. Differences in the composition of preterm and term human milk during early lactation. Pediatr Res 1982;16:113-7.

3. Aquilio E, Spagnoli R, Seri S, et al. Trace element content in human milk during lactation of preterm newborns. Biol Trace Elem Res 1996;51: 63-70.

4. Schanler RJ, Lau C, Hurst NM, et al. Randomized trial of donor human milk vs preterm formula as substitutes for mothers' own milk in the feeding of extremely premature infants. Pediatrics 2005;116:400-6.

5. Alsaweed M, Hartmann PE, Geddes DT, et al. MicroRNAs in breastmilk and the lactating breast: potential immunoprotectors and developmental regulators for the infant and the mother. Int J Environ Res Public Health 2015;12:13981-4020.

6. Weber JA, Baxter DH, Zhang S, et al. The microRNA spectrum in 12 body fluids. Clin Chem 2010;56:1733-41. 


\section{Articles | Carney et al.}

7. Muroya S, Hagi T, Kimura A, et al. Lactogenic hormones alter cellular and extracellular microRNA expression in bovine mammary epithelial cell culture. J Anim Sci Biotechnol 2016;7:8-18.

8. Hassiotou F, Alsaweed M, Savigni D, et al. Profiling of human milk miRNA. FASEB J 2015;29:582-8.

9. Alsaweed M, Lai CT, Hartmann PE, et al. Human milk miRNAs primarily originate from the mammary gland resulting in unique miRNA profiles of fractionated milk. Sci Rep 2016;6:1-13.

10. Zhou Q, Li M, Wang X, et al. Immune-related microRNAs are abundant in breast milk exosomes. Int J Biol Sci 2012;8:118-23.

11. Alsaweed M, Ching TL, Hartmann PE, et al. Human milk cells contain numerous miRNAs that may change with milk removal and regulate multiple physiological processes. Int J Mol Sci 2016;17:956-86.

12. Rottiers V, Näär AM. MicroRNAs in metabolism and metabolic disorders. Nat Rev Mol Cell Biol 2012;13:239-50.

13. Munch EM, Harris RA, Mohammad M, et al. Transcriptome profiling of microRNA by Next-Gen deep sequencing reveals known and novel miRNA species in the lipid fraction of human breast milk. PLoS ONE 2013;8:e50564.

14. Arntz OJ, Pieters BC, Oliveira MC, et al. Oral administration of bovine milk derived extracellular vesicles attenuates arthritis in two mouse models. Mol Nutr Food Res 2015;59:1701-2.

15. Savage JS, Birch LL, Marini M, et al. Effect of the INSIGHT responsive parenting intervention on rapid infant weight gain and overweight status at age 1 year: a randomized clinical trial. JAMA Pediatr 2016;170:742-9.

16. Vlachos IS, Zagganas K, Paraskevopoulou MD, et al. DIANA-miRPath v3. 0: deciphering microRNA function with experimental support. Nucleic Acids Res 2015;43:W460-6.

17. Szklarczyk D, Franceschini A, Wyder S, et al. STRING v10: proteinprotein interaction networks, integrated over the tree of life. Nucleic Acids Res 2015;43:D447-52.

18. Walley AJ, Blakemore AI, Froguel P. Genetics of obesity and the prediction of risk for health. Hum Mol Genet 2006;15:R124-30.

19. Ikeda K, Horie-Inoue K, Ueno T, et al. miR-378a-3p modulates tamoxifen sensitivity in breast cancer MCF-7 cells through targeting GOLT1A. Sci Rep 2015;5:1-12.

20. Gonul O, Aydin HH, Kalmis E, et al. Effects of Ganoderma lucidum (higher basidiomycetes) extracts on the miRNA profile and telomerase activity of the MCF-7 breast cancer cell line. Int J Med Mushrooms 2015;17:231-9.

21. Qin W, Tang Y, Yang N, et al. Potential role of circulating microRNAs as a biomarker for unexplained recurrent spontaneous abortion. Fertil and Steril 2016;105:1247-54.

22. Shah MY, Pan X, Fix LN, et al. 5-fluorouracil drug alters the microrna expression profiles in MCF-7 breast cancer cells. J Cell Physiol 2011;226: 1868-78.
23. Matamala N, Vargas MT, González-Cámpora R, et al. MicroRNA deregulation in triple negative breast cancer reveals a role of miR-498 in regulating BRCA1 expression. Oncotarget 2016;7:20068-79.

24. Zhang K, Zhao S, Wang Q, et al. Identification of micrornas in nipple discharge as potential diagnostic biomarkers for breast cancer. Ann Surg Oncol 2015;22:536-44.

25. Yang F, Zhang W, Shen $Y$, et al. Identification of dysregulated microRNAs in triple-negative breast cancer. Int J Onc 2015;46:927-32.

26. Kumari A. Changes in MicroRNA target Sites Attributed to Single Nucleotide Polymorphisms may Influence Breast Cancer Susceptibility. Doctoral Dissertation, 2014.

27. Maltseva DV, Galatenko VV, Samatov TR, et al. miRNome of inflammatory breast cancer. BMC Res Notes 2014;7:871-1.

28. Lingwood CA. Glycosphingolipid functions. Cold Spring Harb Persp Biol 2011;3:a004788.

29. van Meer G, Lisman Q. Sphingolipid transport: rafts and translocators. J Biol Chem 2002;277:25855-8.

30. Jiang M, Sang X, Hong Z. Beyond nutrients: food-derived microRNAs provide cross-kingdom regulation. Bioessays 2012;34:280-4.

31. Arroyo JD, Chevillet JR, Kroh EM, et al. Argonaute2 complexes carry a population of circulating microRNAs independent of vesicles in human plasma. Proc Natl Acad Sci USA 2011;108:5003-8.

32. Hill PD, Aldag JC, Demirtas H, et al. Association of serum prolactin and oxytocin with milk production in mothers of preterm and term infants. Biol Res Nurs 2009;10:340-9.

33. Ferraro L, Ravo M, Nassa G, et al. Effects of oestrogen on microRNA expression in hormone-responsive breast cancer cells. Horm Cancer 2012;3:65-78.

34. Cochrane DR, Spoelstra NS, Richer JK. The role of miRNAs in progesterone action. Mol Cell Endocrinol 2012;357:50-9.

35. Sethi JK, Hotamisligil GS. The role of TNF $\alpha$ in adipocyte metabolism. Semin Cell Dev Biol 1999;10:19-29.

36. Wang L, Du Z, Liu J, et al. Association of UCP3, APN, and TNF- $\alpha$ Gene polymorphisms with type 2 diabetes in a population of northern Chinese Han patients. Chem Res Chinese Univ 2012;28:255-8.

37. Nisoli E, Tonello C, Landi M, et al. Functional studies of the first selective beta 3-adrenergic receptor antagonist SR 59230A in rat brown adipocytes. Mol Pharmacol 1996;49:7-14.

38. Lee MJ, Fried SK. The glucocorticoid receptor, not the mineralocorticoid receptor, plays the dominant role in adipogenesis and adipokine production in human adipocytes. Int J Obes 2014;38:1228-33.

39. Culpin RE, Proctor SJ, Angus B, et al. A 9-series microRNA signature differentiates between germinal centre and activated B-cell-like diffuse large B-cell lymphoma cell lines. Int J Oncol 2010;37:367-77.

40. Golding J, Emmett PM, Rogers IS. Gastroenteritis, diarrhoea and breast feeding. Early Hum Dev 1997;49:S83-103. 